\title{
Optical spectroscopy of BL Lacertae objects
}

\section{Broad lines, companion galaxies, and redshift lower limits}

\author{
B. Sbarufatti ${ }^{1}$, R. Falomo ${ }^{2}$, A. Treves ${ }^{1}$, and J. Kotilainen ${ }^{3}$ \\ 1 Università dell'Insubria, via Valleggio 11, 22100 Como, Italy \\ e-mail: boris . sbarufatti@uninsubria.it \\ 2 INAF, Osservatorio Astronomico di Padova, Vicolo dell'Osservatorio 5, 35122 Padova, Italy \\ 3 Tuorla Observatory, University of Turku, Väisäläntie 20, 21500 Piikkiö, Finland
}

Received 19 April 2006 / Accepted 11 May 2006

\section{ABSTRACT}

\begin{abstract}
Aims. We present optical spectroscopy for a sample of BL Lac objects, to determine their redshift, to study their broad emission line properties, and to characterize their close environment.

Methods. Twelve objects were observed using the ESO $3.6 \mathrm{~m}$ and NOT $2.5 \mathrm{~m}$ telescopes, obtaining spectra for the BL Lacs and for nearby sources.

Results. For seven of these objects, nuclear emission lines and/or absorption lines from the host galaxy were detected. In all the four cases where absorption lines were revealed, the host galaxy has been resolved with HST or ground-based imaging. The broad $\mathrm{H}_{\alpha}$ luminosities (or their upper limits) of the BL Lacs are similar to those of radio-loud quasars. For two BL Lacs, spectroscopy of close companions indicates that they are at the redshift of the BL Lacs, and therefore physically associated and probably interacting. Five BL Lacs have a featureless spectrum. In these cases, we apply a new technique to derive lower limits for their redshift, which are consistent with the lower limits deduced from imaging.
\end{abstract}

Key words. BL Lacertae objects: general

\section{Introduction}

The BL Lacertae objects (hereafter BL Lacs) are a relatively rare subclass of active galactic nuclei (AGN) characterized by luminous, rapidly variable UV-to-NIR non-thermal continuum emission and polarization, strong compact flat-spectrum radio emission, and superluminal motion. Similar properties are also observed in flat-spectrum radio, quasars and these two types of AGN are often grouped together into the class of blazars. The continuum emission of BL Lacs is boosted by relativistic beaming, which depresses the equivalent width $(E W)$ of the spectral lines. Apart from possible fluorescent emission lines as in other types of AGN, however absorption lines from the stellar population of the host galaxy, from intervening material and from the halo of the Milky Way are expected to be present in BL Lacs. These lines are probes of the physical conditions in the source and in the intervening medium. They are obviously the most direct way of determining or constraining the redshift of the source.

The detection of weak lines requires high $S / N$ spectroscopy that, for most BL Lacs, translates into a necessity to use large telescopes. Until recently, most of the work in this field has made use of 2-4 m class telescopes (e.g. Falomo et al. 1993; Stickel \& Kühr 1993; Véron-Cetty \& Véron 1993; Bade et al. 1994; Falomo et al. 1994; Falomo 1996; Marchã et al. 1996; Drinkwater et al. 1997; Laurent-Muehleisen et al. 1998; Landt et al. 2001; Rector \& Stocke 2001; Londish et al. 2002; Hook et al. 2003; Carangelo et al. 2003).

However, significantly better results have been obtained with $8 \mathrm{~m}$ class telescopes (e.g. Heidt et al. 2004; Sowards-Emmerd et al. 2005; Woo et al. 2005). In particular, a substantial step forward in the detection of faint spectral lines was achieved by our extensive study of 42 BL Lacs performed with the ESO VLT (Sbarufatti et al. 2005a, 2006, hereafter S05a and S06), where we determined the redshift for 18 sources and developed a technique for obtaining redshift lower limits for lineless sources.

In this paper, we complement the ESO-VLT dataset by observations with 2-4 m class telescopes of sources that were not observed with the VLT or that were observed in a different spectral range. The sample of 12 objects was taken from the list of BL Lacs in Padovani \& Giommi (1995) by selecting the brightest targets among those with no available redshift, along with bright, nearby targets to search for broad emission lines (in particular $\mathrm{H}_{\alpha}$ ), and/or to study their environment. The first results of this campaign concerning new redshifts were published by Carangelo et al. (2003). Here we discuss the featureless objects, the search for broad lines, and the properties of companions of the BL Lacs. For the five lineless objects, we apply a technique, developed in S06, that allows us to set lower limits to the redshift. For the four low redshift objects, we report a measurement or an upper limit for the broad component of the $\mathrm{H}_{\alpha}$ emission line. We also present and discuss the spectra of the close companions of $1 \mathrm{H} 0323+022$ and $1 \mathrm{ES} 1440+122$. The following cosmological parameters are adopted throughout this paper: $H_{0}=70 \mathrm{~km} \mathrm{~s}^{-1} \mathrm{Mpc}^{-1}, \Omega_{\Lambda}=0.7, \Omega_{\mathrm{m}}=0.3$.

\section{Observations, data reduction, and analysis}

In Table 1 we report a journal of the observations and in Table 2 the instrumental configurations. The low-resolution setups (A, $\mathrm{B}, \mathrm{N})$ were used for determinating redshifts and for studying 
Table 1. Journal of observations.

\begin{tabular}{lccccccccc}
\hline \hline \multicolumn{1}{c}{$\begin{array}{c}\text { Object } \\
\text { name }\end{array}$} & $\begin{array}{c}\text { RA } \\
(\mathrm{J} 2000)\end{array}$ & $\begin{array}{c}\text { Dec } \\
(\mathrm{J} 2000)\end{array}$ & $z$ & Ref. & $m_{R}{ }^{2}$ & Setup & $S / N$ & $\begin{array}{c}\text { Exposure time } \\
(\mathrm{s})\end{array}$ & Date \\
\hline PKS 0109+224 & 011205 & +224454 & $*$ & & 15.6 & $\mathrm{~B}$ & 230 & 1800 & 26 Jul. 01 \\
1H 0323+022 & 032613 & +022515 & 0.147 & $\mathrm{~F} 86$ & 18.4 & $\mathrm{~A}$ & 30 & 2100 & 15 Jan. 02 \\
& & & & & 18.4 & $\mathrm{C}$ & 30 & 2400 & 13 Jan. 02 \\
PKS 0521-36 & 052257 & -362703 & 0.055 & $\mathrm{D} 79$ & 15.1 & $\mathrm{C}$ & 90 & 1800 & 26 Jul. 01 \\
PKS 0548-322 & 055041 & -321611 & 0.068 & $\mathrm{~F} 76$ & 16.4 & $\mathrm{C}$ & 50 & 1800 & 13 Jan. 02 \\
MS0622.5-5256 & 062337 & -525757 & $*$ & & 19.2 & $\mathrm{~A}$ & 20 & 2400 & 14 Jan. 02 \\
1ES 1106+244 & 110916 & +241120 & $(0.46)^{3}$ & $\mathrm{~S} 05 \mathrm{~b}$ & 18.7 & $\mathrm{~N}$ & 15 & 3600 & 9 Mar. 05 \\
1ES 1239+069 & 124148 & +063601 & $*$ & & 19.2 & $\mathrm{~A}$ & 20 & 2400 & 25 Jul. 01 \\
PG 1437+398 & 143918 & +393242 & $(0.26)^{3}$ & $\mathrm{~S} 05 \mathrm{~b}$ & 16.9 & $\mathrm{~N}$ & 60 & 3600 & 9 Mar. 05 \\
1ES 1440+122 & 144248 & +120040 & 0.162 & $\mathrm{~S} 93$ & 17.2 & $\mathrm{~A}$ & 60 & 2400 & 24 Jul. 01 \\
PKS 2005-489 & 200925 & -484954 & 0.071 & $\mathrm{~F} 87$ & 13.9 & $\mathrm{~A}$ & 400 & 2400 & 25 Jul. 01 \\
& & & & & 13.9 & $\mathrm{C}$ & 250 & 2400 & 25 Jul. 01 \\
PKS 2155-304 & 215851 & -301331 & 0.116 & $\mathrm{~F} 93$ & 13.1 & $\mathrm{~A}$ & 90 & 2400 & 25 Jul. 01 \\
PKS 2201+04 & 220418 & +044002 & 0.027 & $\mathrm{~V} 93$ & 15.2 & $\mathrm{C}$ & 100 & 1800 & 13 Jan. 02 \\
\hline
\end{tabular}

${ }^{1}$ References for redshift determination: F76: Fosbury \& Disney (1976); D79: Danziger et al. (1979); F86: Filippenko et al. (1986); F87: Falomo et al. (1987); S93: Schachter et al. (1993); F93: Falomo et al. (1993); V93: Véron-Cetty \& Véron (1993); S05b: Sbarufatti et al. (2005b).

${ }^{2} R$-band magnitudes determined from the flux measured in our spectra.

${ }^{3}$ The imaging redshift estimate from S05b.

Table 2. Instrumental configurations.

\begin{tabular}{cccccr}
\hline \hline Setup & Telescope & $\begin{array}{c}\text { Slit } \\
(\operatorname{arcsec})\end{array}$ & $\begin{array}{c}\text { Range } \\
(\AA)\end{array}$ & $\begin{array}{c}\text { Disp. } \\
\left(\AA \mathrm{pix}^{-1}\right)\end{array}$ & $\begin{array}{r}\text { Resol. } \\
(\AA)\end{array}$ \\
\hline $\mathbf{A}$ & ESO 3.6 + EFOSC2 & 1.2 & $4085-7520$ & 1.68 & 10.5 \\
$\mathbf{B}$ & ESO 3.6 + EFOSC2 & 1.2 & $5200-9350$ & 2.06 & 12.8 \\
$\mathbf{C}$ & ESO 3.6 + EFOSC2 & 1.0 & $6285-8210$ & 0.95 & 5.9 \\
$\mathbf{N}$ & NOT 2.5 + ALFOSC & 2.5 & $3200-9100$ & 3.00 & 32.4 \\
\hline
\end{tabular}

sources in the close environment of the BL Lacs, while the medium resolution setup (C) was used to search for the broad components of emission lines, especially $\mathrm{H}_{\alpha}$.

Data reduction was performed using IRAF $^{1}$ (Tody 1986, 1993), following the standard procedures for spectral analysis. This includes bias subtraction, flat fielding, and removal of bad pixels. For each target, we obtained two spectra in order to get a good correction of the cosmic rays and to check for the reality of weak spectral features. The individual frames were then combined into a single average image. Wavelength calibration was performed using the spectra of a helium/neon/argon lamp obtained during the same observing night, resulting in an accuracy of $\sim 3 \AA$ (rms). From these calibrated final images, we extracted the one-dimensional spectra adopting an optimal extraction algorithm (Horne 1986) to improve the $S / N$.

Although this program did not require optimal photometric conditions, the sky was clear during most of the observations. This enables us to perform a spectrophotometric calibration of the data using standard stars (Oke 1990) observed during the same nights. The ESO spectral setups $\mathrm{B}$ and $\mathrm{C}$ at wavelengths $\lambda>7000 \AA$ are affected by fringing. This was corrected for using flat field images taken immediately before or after the spectrum of the object, with the telescope in the same position. In the cases where such flat field images were not available, the resulting fringing pattern makes the detection of features in this spectral range very difficult, if not impossible. Finally, all the spectra were dereddened following the extinction law by

${ }^{1}$ IRAF (Image Reduction and Analysis Facility) is distributed by the National Optical Astronomy Observatories, which are operated by the Association of Universities for Research in Astronomy, Inc., under cooperative agreement with the National Science Foundation.
Cardelli et al. (1989) and assuming the $E_{B-V}$ values computed by Schlegel et al. (1998).

In Fig. 1, we present the optical spectrum of each source. In order to show the continuum shape and the faint features more clearly, we report both the flux-calibrated and the normalized spectrum for each object. The main emission and absorption features are identified, while interstellar absorption features are marked as ISM and telluric absorption features as $\oplus$. All these spectra are electronically available in our spectroscopic library of BL Lacs, at http: //www . oapd.inaf.it/zbllac/.

\subsection{Redshift lower limits}

The spectra appear featureless for five objects. In these cases, using the minimum observable $E W\left(E W_{\min }\right)$, it is possible to derive a lower limit for the redshift. This procedure is described in detail in S06. Briefly, it is based on the assumption that the host galaxy is an elliptical with an absolute magnitude of $M_{R} \simeq-22.9 \pm 0.5$, as derived from the analysis of a homogeneous sample of HST images of BL Lacs (see Urry et al. 2000; Sbarufatti et al. 2005b, hereafter S05b). By adopting a template for the spectrum of an elliptical galaxy (Kinney et al. 1996), it was shown by S06 that from the apparent magnitude of the BL Lac, and the $E W_{\min }$ in the spectrum, one can obtain a lower limit to the redshift. The lower limits from this procedure and their comparison with redshifts or lower limits deduced from the imaging of the host galaxy are reported in Table 3.

\section{Results for individual objects}

PKS 0109+224: this radio source was discovered in the $5 \mathrm{GHz}$ Green Bank survey (Davis 1971) and subsequently classified as a BL Lac by Owen \& Muffson (1977). It exhibits significant variability in flux and polarization in both radio and optical bands (Ciprini et al. 2004). The host galaxy was not detected in images obtained at the NTT (Falomo 1996) and the NOT (Nilsson et al. 2003). The claimed detection of the host by Wright et al. (1998) $\left(m_{K}=12.2\right)$ is dubious, since the host is resolved only in one of their two images. Based on the non-detection of the host and assuming that the host has an absolute magnitude $M_{R}=-23.5$, Falomo (1996) proposed a lower limit to its redshift of $z>0.4$. 

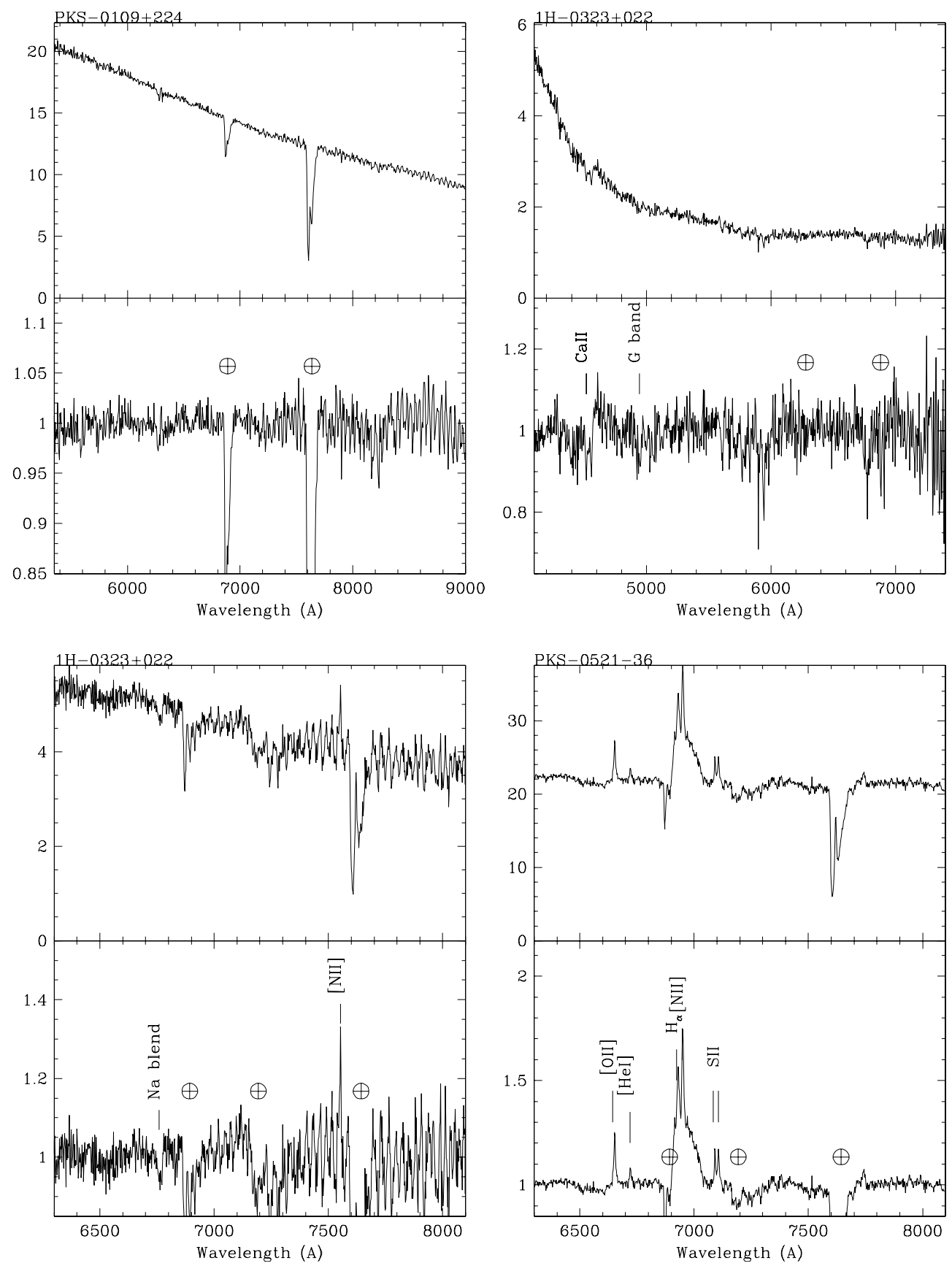

Fig. 1. Spectra of the BL Lacs observed with the ESO $3.6 \mathrm{~m}$ and the NOT $2.5 \mathrm{~m}$ telescopes. Top panel: flux calibrated spectra. Bottom panel: spectra normalized with respect to the continuum. Telluric bands are indicated by $\oplus$, spectral lines are marked by the line ID, and absorption features from atomic species in the interstellar medium of our galaxy are labeled by ISM.

Table 3. Featureless objects.

\begin{tabular}{clcr}
\hline \hline Object name & $E W_{\min }$ & $z_{\min }$ & $z_{\text {ima }}{ }^{1}$ \\
\hline PKS 0109+224 & 0.43 & $>0.18$ & $>0.40$ \\
MS 0622.5-525 & 1.48 & $>0.49$ & $>0.41$ \\
1ES 1106+244 & 2.5 & $>0.29$ & 0.46 \\
1ES 1239+069 & 0.75 & $>0.60$ & $>0.92$ \\
PG 1437+398 & 0.8 & $>0.24$ & 0.26 \\
\hline
\end{tabular}

${ }^{1}$ Imaging redshifts from S05b. For PKS 0109+224, see Falomo (1996).

Previous low-medium $S / N$ optical spectra were featureless (Wills $\&$ Wills 1979; Falomo et al. 1994). Although we reach a high $S / N$
$(S / N=230)$ in the red $(5500-9000 \AA)$, the spectrum remains featureless (Fig. 1). We determine $E W_{\min }=0.43 \AA$, which implies a redshift lower limit of $z>0.18$ according to our adopted procedure, consistent with, but considerably lower than, the limit from imaging.

1H 0323+022: this X-ray selected object (Doxsey et al. 1983) was classified as a BL Lac by Margon \& Jacoby (1984). It exhibits significant optical polarization (Feigelson et al. 1986) and variability (Villata et al. 2000). The host galaxy was resolved in ground-based images by Feigelson et al. (1986), Falomo (1996), and Nilsson et al. (2003). The signature of the host galaxy at $z=0.147$ was also clearly apparent in the optical 

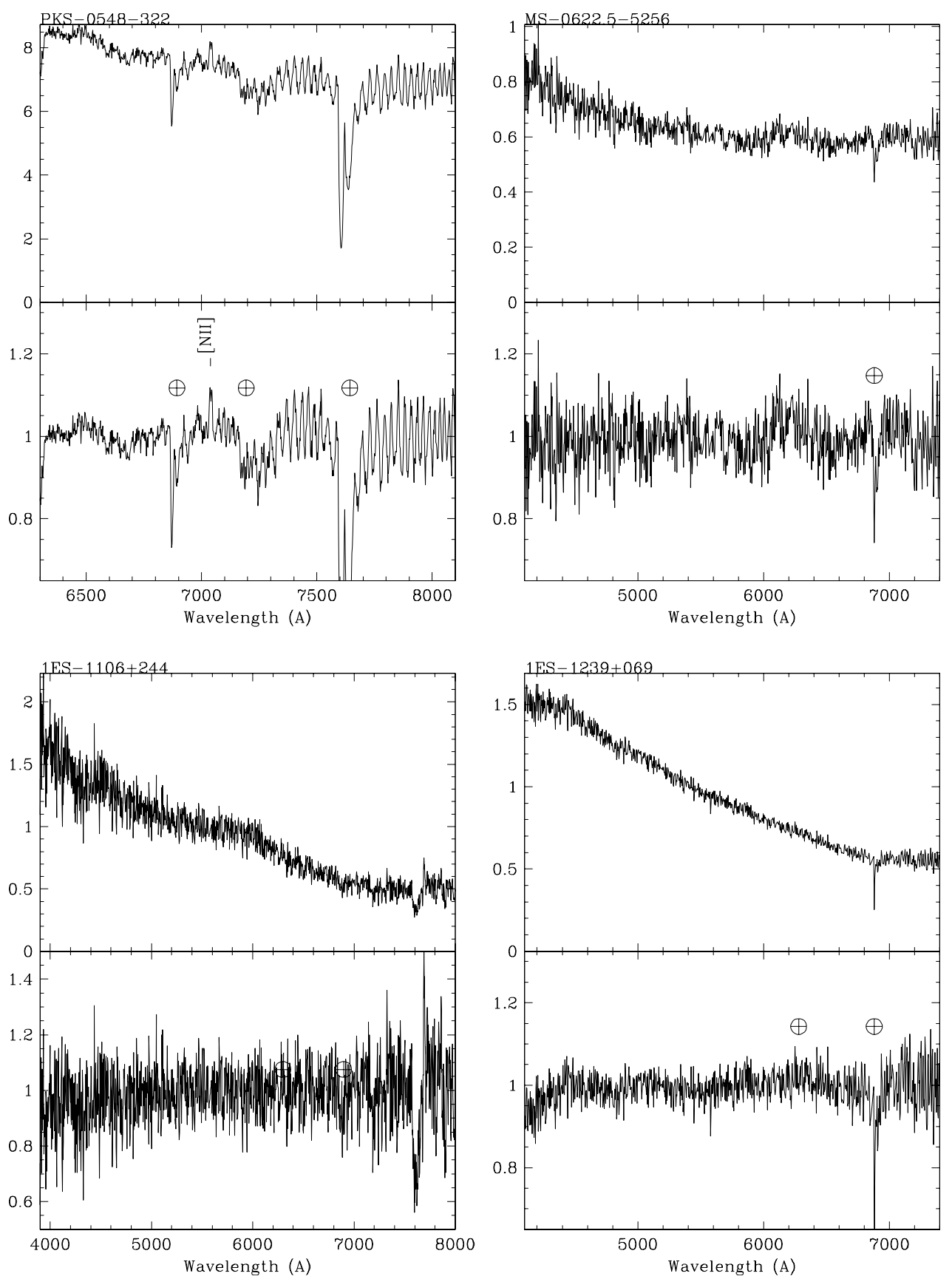

Fig. 1. continued.

spectrum of Filippenko et al. (1986). Despite being dominated by the strong nuclear component, our new spectrum (Fig. 1), clearly shows several absorption lines from the host galaxy, i.e. CaII $\lambda \lambda 3934,3968$ and $\mathrm{G}$ band $\lambda 4305$ (setup A), and NaI $\lambda 5892$ (setup C), confirming its redshift as $z=0.147$. The spectrum also reveals the narrow [NII] $\lambda 6583$ emission line at this redshift. However, no broad $\mathrm{H}_{\alpha}$ emission is detected, with an $E W_{\text {min }}$ limit of $1 \AA$, corresponding to an upper limit of $1.8 \times 10^{40} \mathrm{erg} \mathrm{s}^{-1}$ for the broad $\mathrm{H}_{\alpha}$ luminosity.

This BL Lac is located in a complex environment (e.g. Falomo 1996), as shown in Fig. 2. At a distance of $\sim 1^{\prime}$ east of the BL Lac, there is a bright elliptical galaxy $(\mathrm{G} 1)$ at a similar redshift to the BL Lac $(z \sim 0.16$, Falomo 1996). In the close environment of the BL Lac, there are a number of complex emission features (Fig. 2). In particular, a compact knot-like structure (G2) is located at a distance of $\sim 2.6^{\prime \prime}$. We observed $1 \mathrm{H} 0323+022$ using the setup A, with the slit slightly shifted with respect to the nucleus, to also simultaneously obtain the spectra of G1 and G2 (Fig. 3). G1 is a bright elliptical galaxy at a redshift of $z=0.160$, determined from the $\mathrm{CaII} \mathrm{H} \& \mathrm{~K}, \mathrm{G}$ band, and $\mathrm{MgI}$ absorption lines. Because of its small angular distance from the BL Lac and its low surface brightness, the spectrum of G2 (Fig. 3, second panel) is contaminated by the light from the BL Lac and its host galaxy. Therefore we extracted a spectrum using an identical aperture size to the one used for G2, taken in a position symmetric to G2 with respect to the position of the BL Lac (Fig. 3, third panel), and subtracted it from the spectrum of G2. The decontaminated spectrum of G2 (Fig. 3, bottom panel) has the characteristic shape of an elliptical galaxy, with the absorption features of CaII $\lambda \lambda 3934,3968, \mathrm{G}$ band $\lambda 4305$, and MgI $\lambda 5175$ at $z=0.148$. The measured flux from the spectrum leads to an estimate of $R \simeq 18.6$, which corresponds to $M_{R}=-20.9$. 

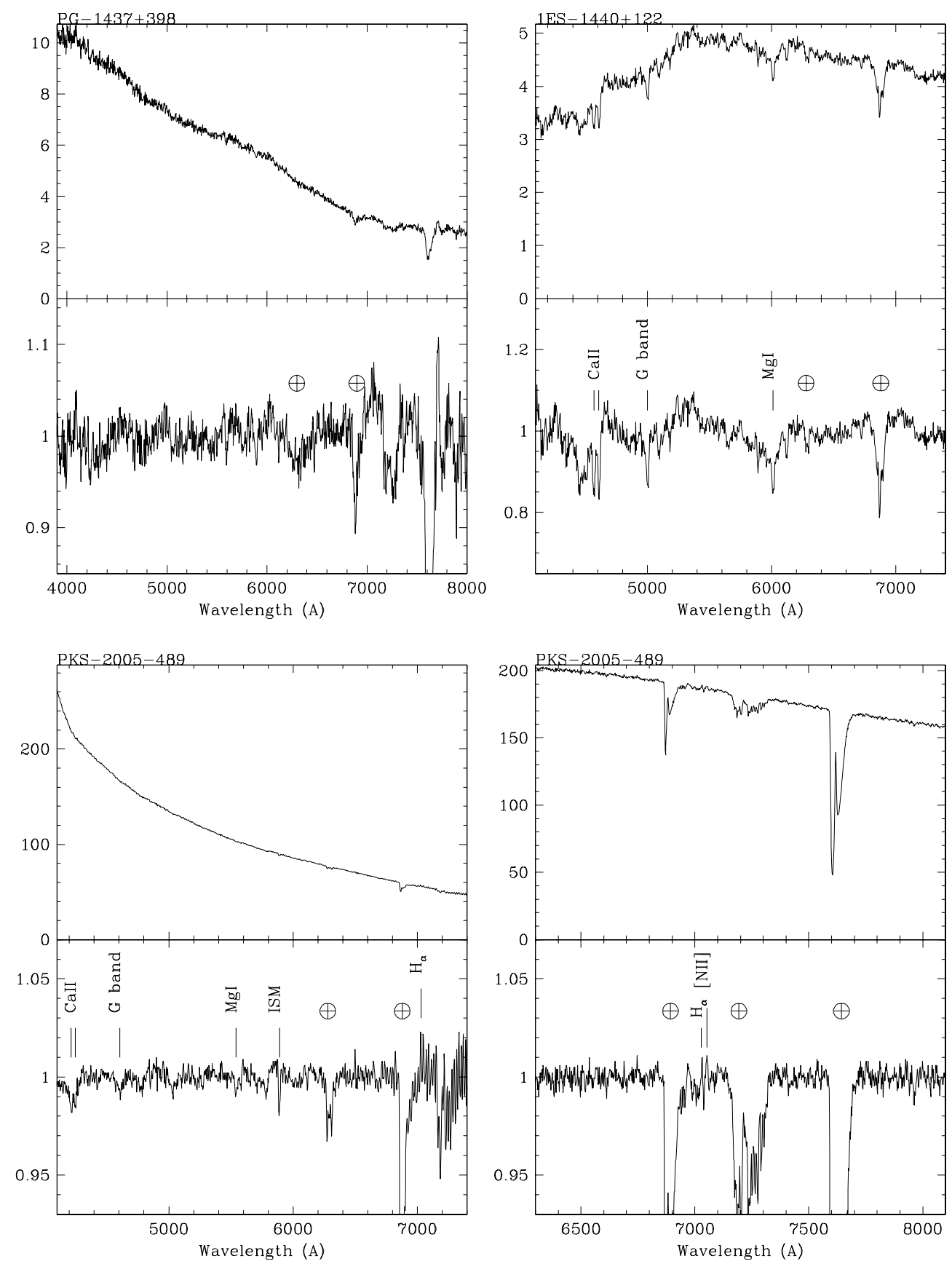

Fig. 1. continued.

Therefore, G2 could be an elliptical dwarf galaxy at the redshift of $1 \mathrm{H} 0323+022$, as already suggested by Falomo (1996). The projected distance of $\mathrm{G} 2$ from the BL Lac is only $d \simeq 8 \mathrm{kpc}$.

PKS 0521-36: this is a well studied BL Lac that has been observed extensively at all wavelengths (see e.g. Pian et al. 1996, and references therein). The host galaxy has been resolved in several imaging studies (e.g. Wurtz et al. 1996; Falomo et al. 1994; Kotilainen et al. 1998; Urry et al. 2000; Cheung et al. 2003). The redshift of this BL Lac $(z=0.055)$ Danziger et al. (1979) is based on both absorption lines from the host galaxy and strong emission lines from the nucleus. In particular, a broad $\left(F W H M \sim 3000 \mathrm{~km} \mathrm{~s}^{-1}\right) \mathrm{H}_{\alpha}$ with varying $E W$ (ranging from 20 to $80 \AA$ ) has been detected (Falomo et al. 1994). Our high $S / N$ spectrum (Fig. 1) clearly shows the narrow [OII] $\lambda 6300$,

HeI $\lambda 5875$, and SII $\lambda \lambda 6716,6730$ emission lines, and the broad $\mathrm{H}_{\alpha}+[\mathrm{NII}]$ blend, with $E W=40.7 \AA$, corresponding to a broad $\mathrm{H}_{\alpha}$ luminosity of $7.5 \times 10^{41} \mathrm{erg} \mathrm{s}^{-1}$, which is within the range previously observed by Falomo et al. (1994).

PKS 0548-322: this X-ray selected BL Lac at $z=0.068$ (Fosbury \& Disney 1976) is located in a rich environment (Falomo et al. 1995). At least one of the companions shows signs of interaction with the BL Lac. The host galaxy has been detected both in imaging (Urry et al. 2000) and in spectroscopy by Falomo \& Ulrich (2000), who did not reveal emission lines. Our new spectrum (Fig. 1) clearly shows a narrow emission line, which we identify as [NII] $\lambda 6583$ at $z=0.068$. The presence of [NII] emission could be a signature of recent star formation in the host galaxy, induced by the interaction with the close 

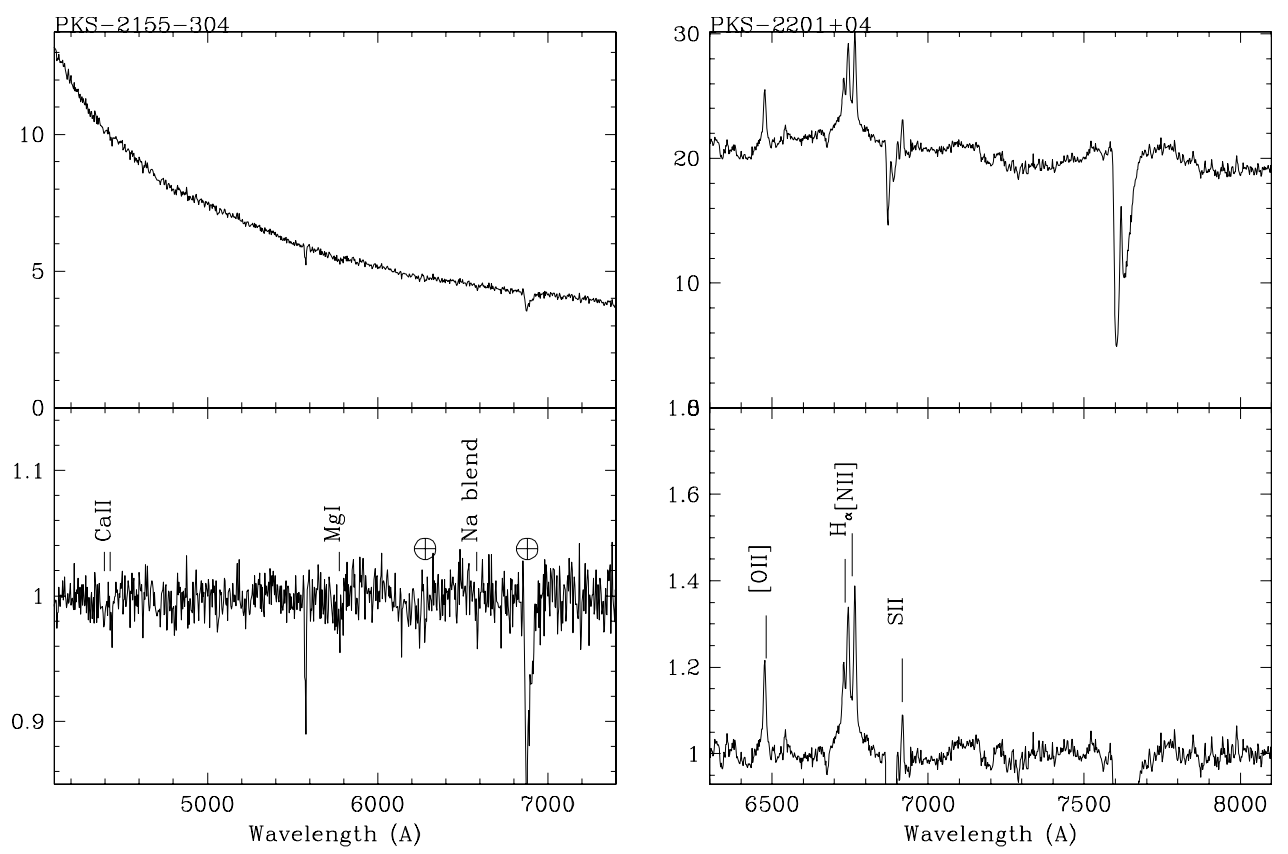

Fig. 1. continued.

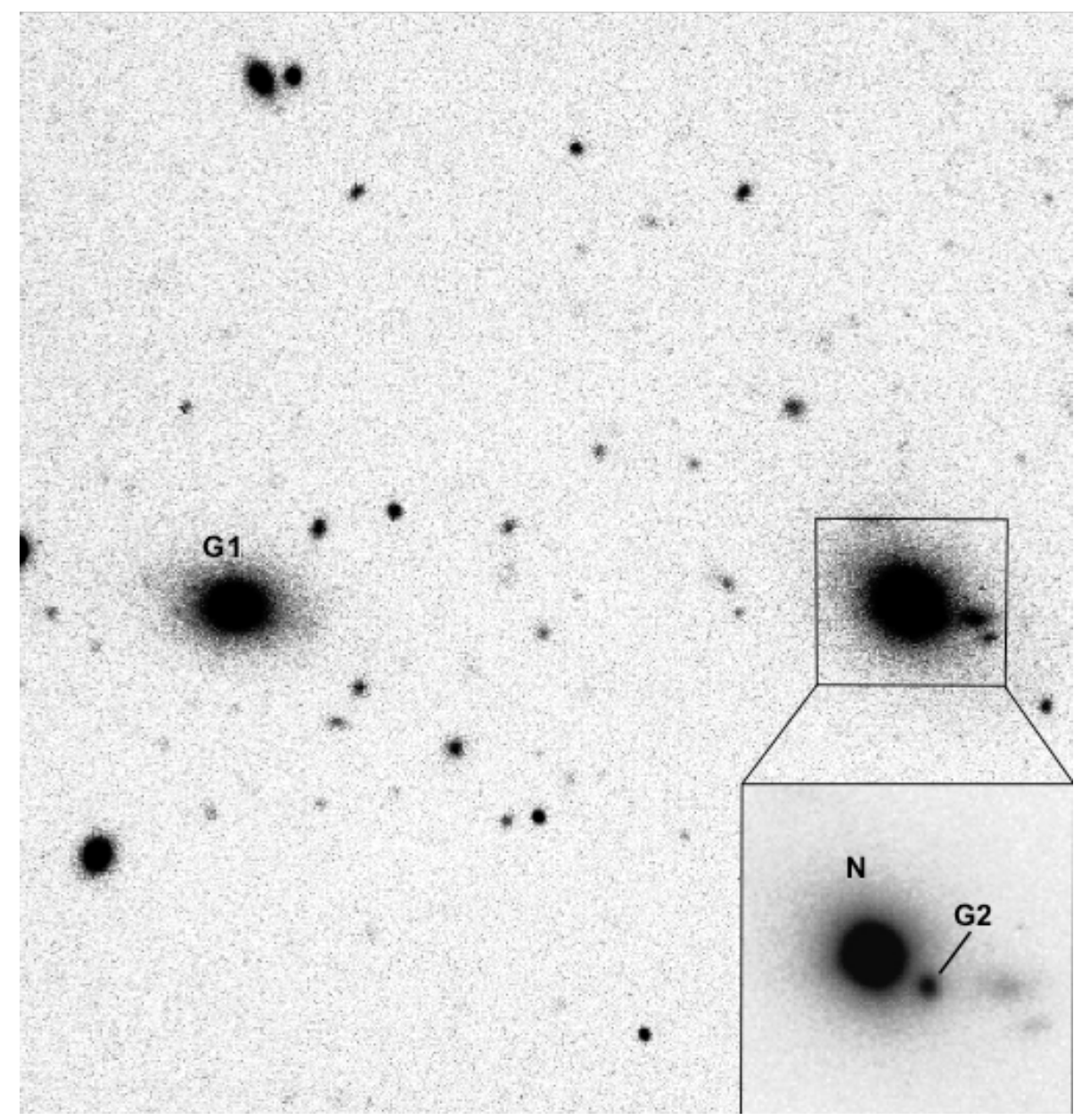

Fig. 2. The environment of $1 \mathrm{H} 0323+22$. In the main panel (ESO $3.6 \mathrm{~m}$ raw image, $10 \mathrm{~s}$ exposure), the companion galaxy G1 is labeled. The subpanel in the lower right hand corner ( $R$-band image, adapted from Falomo 1996) shows a higher resolution image of the BL Lac. The nucleus surrounded by the host galaxy is apparent, together with the compact knot G2. The angular separation between the nucleus and G2 is $2.6^{\prime \prime}$. 


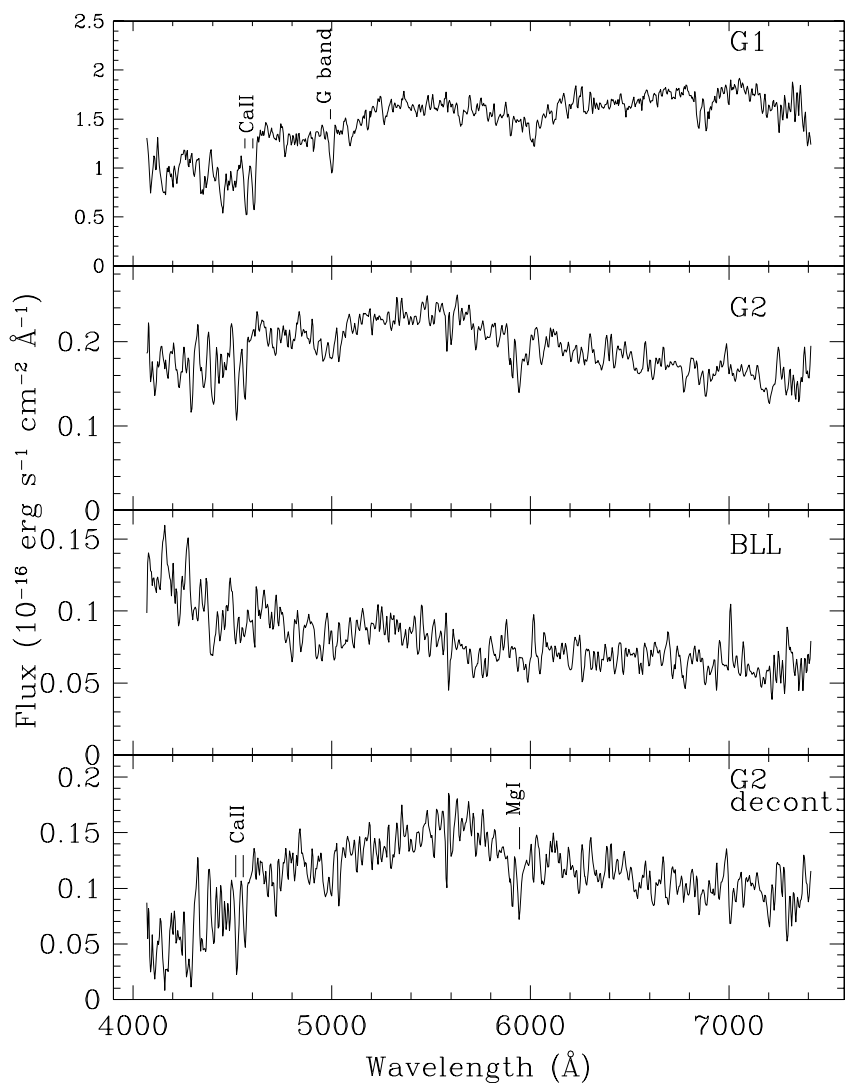

Fig. 3. Spectra of the companion objects of $1 \mathrm{H} 0323+22$. Top panel: G1; second panel: G2 (before decontamination); third panel: spectrum of the BL Lac, taken with an aperture symmetric to G2; bottom panel: G2 (decontaminated spectrum).

companion Falomo et al. (1995). No other emission features are detected, and the $E W_{\text {min }}$ limit of $0.9 \AA$ corresponds to an upper limit of $8.4 \times 10^{39} \mathrm{erg} \mathrm{s}^{-1}$ for the broad $\mathrm{H}_{\alpha}$ luminosity, i.e. five times smaller than the upper limit by Falomo \& Ulrich (2000).

MS0622.5-5256: this is an X-ray selected BL Lac belonging to the EINSTEIN Extended Medium Sensitivity Survey (EMSS, Gioia et al. 1990). Previous low $S / N$ spectroscopy (Stocke et al. 1985) showed a featureless continuum. The HST imaging of this BL Lac (Scarpa et al. 2000a) failed to resolve the host galaxy, suggesting a redshift lower limit $z>0.4$ (see S05b). Our new spectrum (Fig. 1) is featureless with $E W_{\text {min }}=1.48 \AA$, which gives a spectroscopic redshift lower limit of $z>0.49$, very consistent with the lower limit from imaging.

1ES 1106+244: this BL Lac belongs to the EMSS. The host galaxy has been resolved both in ground-based (Falomo \& Kotilainen 1999) and in HST imaging (Urry et al. 2000), indicating a redshift lower limit of $z \simeq 0.42$. Earlier spectroscopy of this BL Lac by Perlman et al. (1996) showed a featureless spectrum. From our moderate $S / N$ spectrum (Fig. 1), we determine $E W_{\text {min }}=2.50 \AA$, which implies a redshift lower limit of $z>0.29$, consistent with the limit obtained from imaging.

1ES 1239+069: this high-energy peaked BL Lac (HBL) has been proposed as a candidate $\mathrm{TeV}$ source by Stecker et al. (1996), being supposedly at a relatively low redshift $(z=0.150$, Perlman et al. 1996). However, this redshift estimate, based on the possible detection of absorption features from the host galaxy, is ruled out by our new spectrum (Fig. 1), from which the measured $E W_{\min }=0.75 \AA$ implies $z>0.60$. Moreover, the nondetection of the host galaxy in imaging sets a further lower limit of $z>0.92$ (S05b), considerably higher than the spectroscopic one, making the detection of this BL Lac in the TeV domain unlikely. Indeed Horan et al. (2004) failed to detect this BL Lac using the Whipple $10 \mathrm{~m} \gamma$-ray telescope.

PG 1437+398: this HBL belongs to the Sedentary Survey (Giommi et al. 2005). Its host galaxy was resolved with HST imaging, giving an imaging redshift of $z \simeq 0.26$ (S05b). Previous optical spectroscopy (Laurent-Muehleisen et al. 1998; White et al. 2000; Scarpa et al. 1995) has shown a featureless spectrum. Note that the redshift $z=0.34$ reported for this BL Lac by the NASA Extragalactic Database is based on a very low $S / N(\sim 5)$ Sloan Digital Sky Survey spectrum ${ }^{2}$. This redshift is ruled out by our new, much higher $S / N(S / N \sim 60)$ spectrum (Fig. 1), which shows a featureless continuum. From the $E W_{\min }$ value $\left(E W_{\min }=\right.$ $0.8 \AA$ ), we deduce a redshift lower limit of $z>0.24$, consistent with the imaging redshift estimate.

1ES 1440+122: this is an X-ray selected BL Lac belonging to the Einstein Slew Survey. It is located in a rich environment (Fig. 4), being surrounded by 20 galaxies (Heidt et al. 1999), thus suggesting that this BL Lac is located in a group or a small cluster of galaxies. The host galaxy has been resolved in several imaging studies (Heidt et al. 1999; Falomo \& Kotilainen 1999; Urry et al. 2000; Kotilainen \& Falomo 2004). High-resolution HST imaging by Scarpa et al. (1999) revealed a very close companion $\left(\sim 0.3^{\prime \prime}\right)$ to this BL Lac, suggesting the possibility of gravitational lensing. This hypothesis was, however, ruled out by a radio-optical study by Giovannini et al. (2004), who demonstrated that the companion object is a foreground star. We obtained spectra of the BL Lac itself (Fig. 1), of a galaxy at a distance of $25^{\prime \prime}(\mathrm{G} 1)$, and of a close companion at a distance of $\sim 2^{\prime \prime}$ (G2; Fig. 5). The spectrum of 1ES1440+122 is dominated by emission from the host galaxy, but the contribution of the nucleus becomes apparent towards blue wavelengths (indeed, the strength of the CaII break is only $\sim 20 \%$ ). The redshift of this BL Lac, $z=0.162$, measured from the CaII $\lambda \lambda 3934,3968$, $\mathrm{G}$ band $\lambda 4305$, and $\mathrm{MgI} \lambda 5175$ absorption lines from the host galaxy confirms the result by Schachter et al. (1993). G1 is a typical elliptical galaxy at a redshift of $z=0.164$ and at a projected distance from the BL Lac of $\sim 98 \mathrm{kpc}$. The other companion, G2, is also an elliptical galaxy at a redshift of $z=0.161$ (see also Nilsson et al. 2003, who discuss their unpublished spectrum of $\mathrm{G} 2$ ), and at a projected distance from the BL Lac of only $\sim 8 \mathrm{kpc}$. Such a small separation strongly indicates that the BL Lac and the galaxy G2 are interacting.

PKS 2005-489: this X-ray selected BL Lac is at low redshift ( $z=0.071$ by Falomo et al. 1987, based on the detection of $\mathrm{H}_{\alpha}$ and [NII] emission lines). More recent spectroscopy has revealed weak NI $\lambda 1135$ and CIII $\lambda 1176$ emission lines from the nucleus (Penton et al. 2004) and absorption lines from the host galaxy (Pesce et al. 1994). The host galaxy of this BL Lac, resolved in several optical and NIR imaging studies (Stickel \& Kühr 1993;

\footnotetext{
2 See Sloan Digital Sky Survey Data Release 4, (http://cas.sdss.org/astro/en/tools/getimg/spectra.asp), plate $1350 / 52786$, fiber 333, and Richards et al. (2002) for a description of the quasar survey.
} 


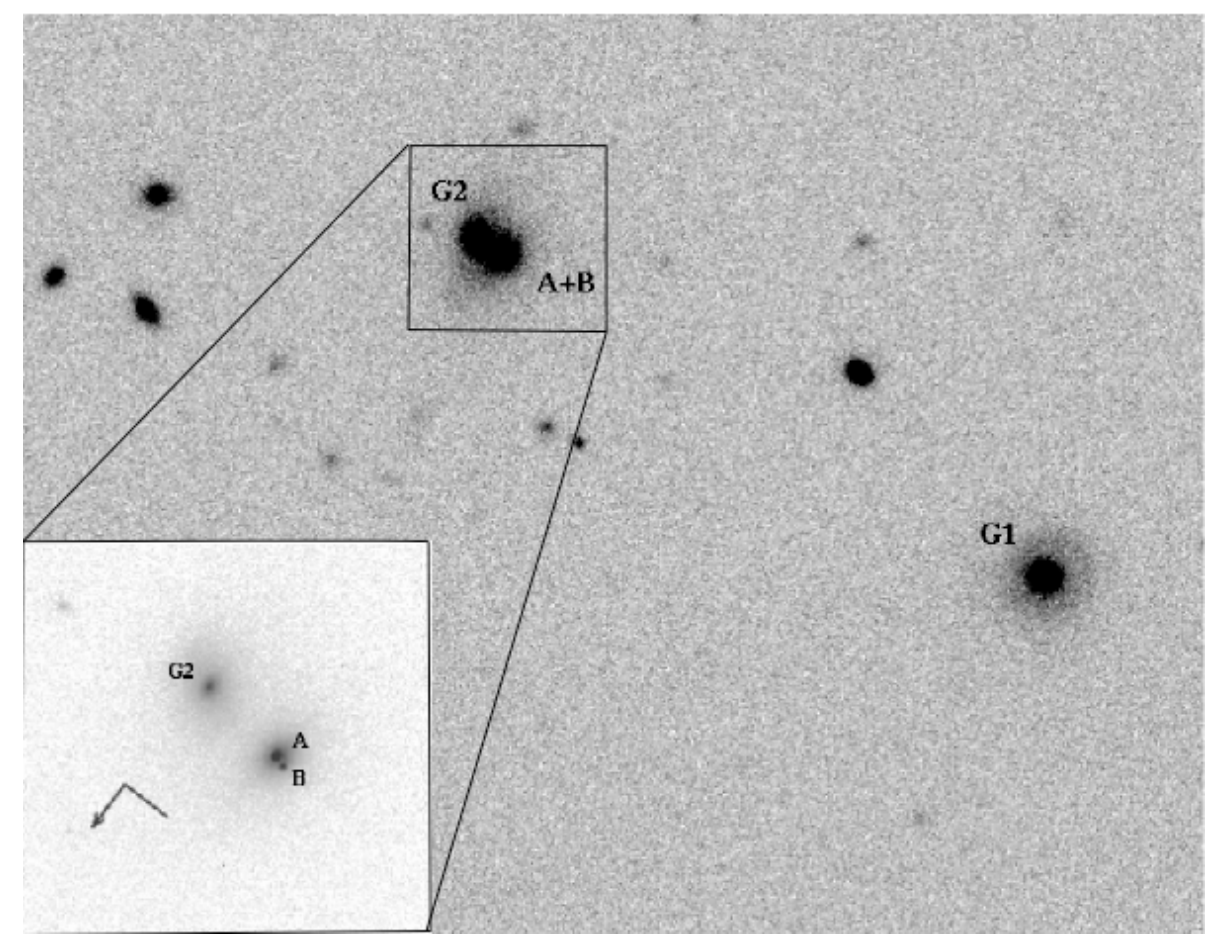

Fig. 4. The environment of 1ES 1440+122. In the main panel ( $R$-band image taken at the NOT by R. Falomo), the locations of galaxies G1 and G2 and the nucleus $(\mathrm{A}+\mathrm{B})$ are indicated. Subpanel: HST detail of the region around the BL Lac (filter F702W image from Scarpa et al. 1999); A is the BL Lac nucleus, B is a foreground star (see Giovannini et al. 2004). The angular separation between A and G2 is $2^{\prime \prime}$. North and East directions are indicated by the arrow.

Falomo 1996; Kotilainen et al. 1998; Urry et al. 2000; Cheung et al. 2003), is a giant elliptical with $M_{R}=-23.1$ in a relatively rich environment (Pesce et al. 1994). Several of the nearby galaxies are known to be at the redshift of the BL Lac (Stickel \& Kühr 1993; Pesce et al. 1994). The optical spectrum of this BL Lac is strongly dominated by the nuclear continuum (Falomo et al. 1994; Perlman et al. 1996). The very high $S / N$ reached by our new spectrum ( 400, setup A; Fig. 1) allowed the detection of the spectroscopic signatures from the host galaxy. The CaII $\lambda \lambda 3934,3968, \mathrm{G}$ band $\lambda 4305$ and $\mathrm{MgI} \lambda 5175$ absorption lines have $E W$ ranging from 0.2 to $0.4 \AA$. The spectrum obtained with setup C confirms the presence of $\mathrm{H}_{\alpha} \lambda 6563$ and [NII] $\lambda 6583$ narrow emission lines. No broad component of $\mathrm{H}_{\alpha}$ is detected, with an $E W_{\text {min }}$ limit of $0.2 \AA$, corresponding to an upper limit of $1.9 \times 10^{41} \mathrm{erg} \mathrm{s}^{-1}$ for the broad $\mathrm{H}_{\alpha}$ luminosity. For recent spectroscopic results on the host galaxy, see Bressan et al. (2006).

PKS 2155-304: although this HBL, a prototype of its class, has been studied in a large number of papers at all wavelengths (e.g. Pesce et al. 1997, and references therein), little optical spectroscopy has been published. Its redshift, $z=0.116$, has been measured by Falomo et al. (1993), from the $\mathrm{G}$ band and the MgI and $\mathrm{NaI}$ absorption lines (and a marginally detected CaII doublet) in a spectrum of the host galaxy with the slit offset from the nucleus. The host galaxy has been resolved in imaging studies, with $I=14.8$ (Falomo et al. 1991; Kotilainen et al. 1998), consistent with this redshift. Our new high $S / N$ ESO spectrum (Fig. 1), also taken with the slit offset from the nucleus, allows us to confirm detection of all the features reported by Falomo et al. (1993). In particular, the CaII lines $\lambda \lambda 3934,3968$ are clearly revealed, with $E W$ of 0.5 and $0.4 \AA$ for the $\mathrm{K}$ and $\mathrm{H}$ lines, respectively.

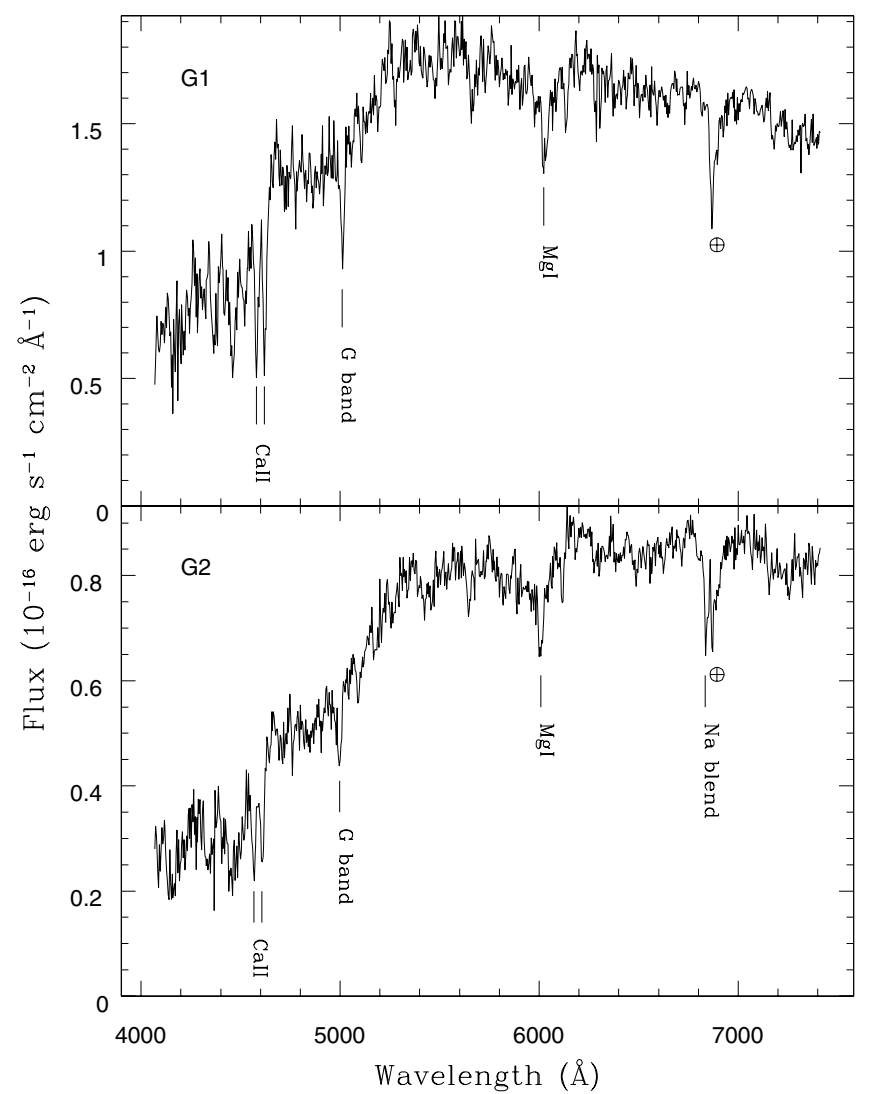

Fig. 5. Spectra of the companion galaxies G1 (top) and G2 (bottom) of $1 \mathrm{ES} 1440+122$. 
PKS 2201+04: this radio source was classified as a BL Lac by Weiler \& Johnston (1980). Its redshift, $z=0.026$, proposed by Wills \& Wills (1976) has been confirmed by more recent observations, showing absorption lines from the host galaxy, along with narrow and broad nuclear emission lines, in particular, broad components of $\mathrm{H}_{\beta}$ and $\mathrm{H}_{\alpha}$ (Falomo et al. 1987; Véron-Cetty \& Véron 1993). Our new spectrum (Fig. 1) reveals the [OII] $\lambda 6300,[\mathrm{NII}] \lambda 6583, \mathrm{H}_{\alpha} \lambda 6563$ and SII $\lambda 6730$ emission lines. The $\mathrm{H}_{\alpha^{-}}$- $\left.\mathrm{NII}\right]$ blend clearly shows the presence of a broad component, with an $E W$ of $13.8 \AA$, corresponding to a broad $\mathrm{H}_{\alpha}$ luminosity of $6.1 \times 10^{40} \mathrm{erg} \mathrm{s}^{-1}$.

\section{Conclusions}

We have presented new, high quality optical spectroscopy of a sample of $12 \mathrm{BL}$ Lac objects. Absorption lines from the host galaxy were detected in four objects. In all these cases, the host galaxy was also resolved in imaging, either with HST (1ES $1440+122$, Urry et al. 2000) or from the ground (1H 0323+022, PKS 2005-489, PKS 2155-304, see Feigelson et al. 1986; Pesce et al. 1994; Kotilainen et al. 1998, respectively). The absolute magnitude of the host is close to $M_{R}=$ -22.9 in all cases, which is typical of BL Lac hosts (S05b). The characterization of the absorption lines from the host galaxy is, however, arduous in most cases, because the $E W$ of the lines is strongly reduced by the beamed non-thermal continuum.

The broad emission-line intensities of BL Lacs are similar to those of radio-loud quasars (e.g. Pian et al. 2005). The two cases where broad $\mathrm{H}_{\alpha}$ is observed in this study (PKS 0521-365, $L_{\mathrm{H}_{\alpha}} \simeq 7.5 \times 10^{41} \mathrm{erg} \mathrm{s}^{-1} ;$ PKS $2201+04, L_{\mathrm{H}_{\alpha}} \simeq 6.1 \times 10^{40} \mathrm{erg} \mathrm{s}^{-1}$ ) confirm this conclusion. It is also consistent with the derived upper limits in the cases of $1 \mathrm{H} 0323+022\left(L_{\mathrm{H}_{\alpha}}<1.8 \times 10^{40} \mathrm{erg} \mathrm{s}^{-1}\right)$, PKS 0548-322 $\left(L_{\mathrm{H}_{\alpha}}<8 \times 10^{39} \mathrm{erg} \mathrm{s}^{-1}\right)$, and PKS 2005-489 $\left(L_{\mathrm{H}_{\alpha}}<1.9 \times 10^{41} \mathrm{erg} \mathrm{s}^{-1}\right)$.

For two BL Lacs (1H 0323+022 and 1ES 1440+122), we have demonstrated that very nearby (projected distance $\sim 8 \mathrm{kpc}$ ) companion galaxies are at the redshift of the BL Lac, indicating that there is a physical association and a likely interaction. Similar cases have been found previously for a number of other BL Lacs (see Pesce et al. 1995; Falomo 1996; Falomo et al. 2000).

Finally, we consider the five BL Lacs that remain featureless in our spectra. Their redshift lower limits, based on the minimum observable $E W$ of the non-detected absorption lines, appear consistent with the ones deduced from imaging. As already noted by $\mathrm{S} 05 \mathrm{~b}$, the imaging technique is more stringent for brighter objects, but the spectral technique is the only available method for faint $\left(m_{V} \geq 18\right)$ sources.

\section{References}

Bade, N., Fink, H. H., \& Engels, D. 1994, A\&A, 286, 381

Bressan, A., Falomo, R., Valdés, J. R., \& Rampazzo, R. 2006, ApJ, 645, L101 Carangelo, N., Falomo, R., Kotilainen, J., Treves, A., \& Ulrich, M.-H. 2003, A\&A, 412, 651

Cardelli, J. A., Clayton, G. C., \& Mathis, J. S. 1989, ApJ, 345, 245

Cheung, C. C., Urry, C. M., Scarpa, R., \& Giavalisco, M. 2003, ApJ, 599, 155

Ciprini, S., Tosti, G., Teräsranta, H., \& Aller, H. D. 2004, MNRAS, 348, 1379

Danziger, I. J., Fosbury, R. A. E., Goss, W. M., \& Ekers, R. D. 1979, MNRAS, 188,415

Davis, M. M. 1971, AJ, 76, 980

Doxsey, R., Bradt, H., McClintock, J., et al. 1983, ApJ, 264, L43

Drinkwater, M. J., Webster, R. L., Francis, P. J., et al. 1997, MNRAS, 284, 85

Falomo, R. 1996, MNRAS, 283, 241
Falomo, R., \& Kotilainen, J. K. 1999, A\&A, 352, 85

Falomo, R., \& Ulrich, M.-H. 2000, A\&A, 357, 91

Falomo, R., Maraschi, L., Treves, A., \& Tanzi, E. G. 1987, ApJ, 318, L39

Falomo, R., Giraud, E., Melnick, J., et al. 1991, ApJ, 380, L67

Falomo, R., Bersanelli, M., Bouchet, P., \& Tanzi, E. G. 1993, AJ, 106, 11

Falomo, R., Scarpa, R., \& Bersanelli, M. 1994, ApJS, 93, 125

Falomo, R., Pesce, J. E., \& Treves, A. 1995, ApJ, 438, L9

Falomo, R., Scarpa, R., Treves, A., \& Urry, C. M. 2000, ApJ, 542, 731

Feigelson, E. D., Bradt, H., McClintock, J., et al. 1986, ApJ, 302, 337

Filippenko, A. V., Djorgovski, S., Spinrad, H., \& Sargent, W. L. W. 1986, AJ, 91, 49

Fosbury, R. A. E., \& Disney, M. J. 1976, ApJ, 207, L75

Gioia, I. M., Maccacaro, T., Schild, R. E., et al. 1990, ApJS, 72, 567

Giommi, P., Piranomonte, S., Perri, M., \& Padovani, P. 2005, A\&A, 434, 385

Giovannini, G., Falomo, R., Scarpa, R., Treves, A., \& Urry, C. M. 2004, ApJ, 613,747

Heidt, J., Nilsson, K., Sillanpää, A., Takalo, L. O., \& Pursimo, T. 1999, A\&A, 341,683

Heidt, J., Tröller, M., Nilsson, K., et al. 2004, A\&A, 418, 813

Hook, I. M., Shaver, P. A., Jackson, C. A., Wall, J. V., \& Kellermann, K. I. 2003, A\&A, 399, 469

Horan, D., Badran, H. M., Bond, I. H., et al. 2004, ApJ, 603, 51

Horne, K. 1986, PASP, 98, 609

Kotilainen, J. K., \& Falomo, R. 2004, A\&A, 424, 107

Kotilainen, J. K., Falomo, R., \& Scarpa, R. 1998, A\&A, 336, 479

Kinney, A. L., Calzetti, D., Bohlin, R. C., et al. 1996, ApJ, 467, 38

Landt, H., Padovani, P., Perlman, E. S., et al. 2001, MNRAS, 323, 757

Laurent-Muehleisen, S. A., Kollgaard, R. I., Ciardullo, R., et al. 1998, ApJS, 118,127

Londish, D., Croom, S. M., Boyle, B. J., et al. 2002, MNRAS, 334, 941

Marchã, M. J. M., Browne, I. W. A., Impey, C. D., \& Smith, P. S. 1996, MNRAS, 281,425

Margon, B., \& Jacoby, G. H. 1984, ApJ, 286, L31

Nilsson, K., Pursimo, T., Heidt, J., et al. 2003, A\&A, 400, 95

Oke, J. B. 1990, AJ, 99, 1621

Owen, F. N., \& Muffson, S. L. 1977, AJ, 82, 776

Padovani, P., \& Giommi, P. 1995a, MNRAS, 277, 1477

Pian, E., Falomo, R., Ghisellini, G., et al. 1996, ApJ, 459, 169

Pian, E., Falomo, R., \& Treves, A. 2005, MNRAS, 361, 919

Penton, S. V., Stocke, J. T., \& Shull, J. M. 2004, ApJS, 152, 29

Perlman, E. S., Stocke, J. T., Schachter, J. F., et al. 1996, ApJS, 104, 251

Pesce, J. E., Falomo, R., \& Treves, A. 1994, AJ, 107, 494

Pesce, J. E., Falomo, R., \& Treves, A. 1995, AJ, 110, 1554

Pesce, J. E., Urry, C. M., Maraschi, L., et al. 1997, ApJ, 486, 770

Rector, T. A., \& Stocke, J. T. 2001, AJ, 122, 565

Richards, G. T., Fan, X., Newberg, H. J., et al. 2002, AJ, 123, 2945

Sbarufatti, B., Treves, A., Falomo, R., et al. 2005a, AJ, 129, 599 (S05a)

Sbarufatti, B., Treves, A., \& Falomo, R. 2005b, ApJ, 635, 173 (S05b)

Sbarufatti, B., Treves, A., Falomo, R., et al. 2006, AJ, 132, 1 (S06)

Scarpa, R., Falomo, R., \& Pian, E. 1995, A\&A, 303, 730

Scarpa, R., Urry, C. M., Falomo, R., \& Treves, A. 1999, ApJ, 526, 643

Scarpa, R., Urry, C. M., Falomo, R., Pesce, J. E., \& Treves, A. 2000, ApJ, 532, 740

Schachter, J. F., Stocke, J. T., Perlman, E., et al. 1993, ApJ, 412, 541

Schlegel, D. J., Finkbeiner, D. P., \& Davis, M. 1998, ApJ, 500, 525

Sowards-Emmerd, D., Romani, R. W., Michelson, P. F., Healey, S. E., \& Nolan, P. L. 2005, ApJ, 626, 95

Stecker, F. W., de Jager, O. C., \& Salamon, M. H. 1996, ApJ, 473, L75

Stickel, M., \& Kühr, H. 1993, A\&AS, 100, 395

Stocke, J. T., Liebert, J., Schmidt, G., et al. 1985, ApJ, 298, 619

Tody, D. 1986, Proc. SPIE, 627, 733

Tody, D. 1993, Astronomical Data Analysis Software and Systems II, 2, ASP Conf. Ser., 52, 173

Urry, C. M., Scarpa, R., O’Dowd, M., et al. 2000, ApJ, 532, 816

Véron-Cetty, M.-P., \& Véron, P. 1993, A\&AS, 100, 521

Villata, M., Raiteri, C. M., Popescu, M. D., et al. 2000, A\&AS, 144, 481

Weiler, K. W., \& Johnston, K. J. 1980, MNRAS, 190, 269

White, R. L., Becker, R. H., Gregg, M. D., et al. 2000, ApJS, 126, 133

Wills, D., \& Wills, B. J. 1976, ApJS, 31, 143

Wills, B. J., \& Wills, D. 1979, ApJS, 41, 689

Woo, J.-H., Urry, M. C., van der Marel, R. P., Lira, P., \& Maza, J. 2005, ApJ, 631,762

Wright, S. C., McHardy, I. M., Abraham, R. G., \& Crawford, C. S. 1998, MNRAS, 296, 961

Wurtz, R., Stocke, J. T., \& Yee, H. K. C. 1996, ApJS, 103, 109 